\title{
Efficient simulation of autofluorescence effects in microscopic lenses
}

\author{
Gross, Herbert; Rodenko, Olga; Esslinger, Moritz; Tünnermann, Andreas
}

Published in:

Proceedings of SPIE

Link to article, DOI:

$10.1117 / 12.2191260$

Publication date:

2015

Document Version

Publisher's PDF, also known as Version of record

Link back to DTU Orbit

Citation $(A P A)$ :

Gross, H., Rodenko, O., Esslinger, M., \& Tünnermann, A. (2015). Efficient simulation of autofluorescence effects in microscopic lenses. In Proceedings of SPIE (Vol. 9626). [962609] SPIE - International Society for Optical Engineering. Proceedings of SPIE - The International Society for Optical Engineering https://doi.org/10.1117/12.2191260

\section{General rights}

Copyright and moral rights for the publications made accessible in the public portal are retained by the authors and/or other copyright owners and it is a condition of accessing publications that users recognise and abide by the legal requirements associated with these rights.

- Users may download and print one copy of any publication from the public portal for the purpose of private study or research.

- You may not further distribute the material or use it for any profit-making activity or commercial gain

- You may freely distribute the URL identifying the publication in the public portal 


\title{
Efficient Simulation of Autofluorescence Effects in Microscopic Lenses
}

\author{
Herbert Gross $^{\mathrm{a}^{*}}$, Olga Rodenko ${ }^{\mathrm{b}}$, Moritz Esslinger ${ }^{\mathrm{c}}$, Andreas Tünnermann ${ }^{\mathrm{a}}$ \\ ${ }^{a}$ Friedrich-Schiller-University Jena, Institute of Applied Physics, Albert-Einstein-Straße 15, Jena, \\ Germany, 07745, ${ }^{\mathrm{b}}$ DTU Fotonik, Department of Photonics Engineering, Technical University of \\ Denmark, Frederiksborgvej 399, 4000 Roskilde, Denmark, ${ }^{c}$ Fraunhofer-Institut für Angewandte \\ Optik und Feinmechanik, Albert-Einstein-Straße 7, 07745 Jena, Germany, presently at Multiphoton \\ Optics GmbH, Building C, Friedrich-Bergius-Ring 15, 97076 Würzburg, Germany
}

\begin{abstract}
The use of fluorescence in microscopy is a well known technology today. Due to the autofluorescence of the materials of the optical system components, the contrast of the images is degraded. The calculation of autofluorescense usually is performed by brute force methods as volume scattering. The efficiency of calculations in this case is extremely low and a huge number of rays must be calculated. In stray light calculations the concept of important sampling is used to reduce computational effort. The idea is to calculate only rays, which have the chance to reach the target surface. The fluorescence conversion can be considered to be a scatter process and therefore a modification of this idea is used here. The reduction factor is calculated by simply comparing in every z-plane of the lenses the size of the illuminated phase space domain with the corresponding acceptance domain. The boundaries of the domains are determined by simple tracing of the limiting rays of the light cone of the source as well as the pixel area under consideration. The small overlap of both domains can be estimated by geometrical considerations. The correct photometric scaling and the discretization of the volumes must be performed properly. Some necessary approximations produce negligible errors. The improvement in run time is in the range of $10^{4}$. It is shown with some practical examples of microscopic lenses, that the results are comparable with conventional methods. The limitations and the consequences for questions of the lens design are discussed.
\end{abstract}

Keywords: Microscopy, autofluorescence, straylight, contrast, simulation

\section{INTRODUCTION}

Fluorescence microscopy is one of the most common imaging techniques in biology and biochemistry ${ }^{1-3}$. It has an outstanding sensitivity, allowing for detection schemes down to the single molecular level ${ }^{4}$. Fluorescence microscopy has found many applications due to variety of fluorophores and excitation wavelengths that can be used.

When observing single molecules or other weakly interacting samples, the background noise level in the imaging setup strongly impacts the signal-to-noise ratio (SNR). Common sources of optical noise include unwanted emission by autofluorescent cells or tissues, emission of fluorophores which are not bound to target of interest and autofluorescence produced by a microscope itself. Several methods can be employed during measurement to reduce autofluorescence which originates from the sample ${ }^{5-8}$. There are theoretical models able to predict fluorescence from the sample ${ }^{9}$. Mathematical methods, in which the background fluorescence is subtracted from the image, can be used in postprocessing ${ }^{10}$. A part of the fluorescence background may be emitted by materials employed in microscope objective lenses. Optical glasses, cementing, immersion media and glue may absorb the excitation light in the near UV range and re-emit autofluorescent light. Especially optical glasses have to be chosen with consideration for fluorescence microscopy. It is known that lead glasses exhibit autofluorescence in the near UV range ${ }^{11}$. Figure 1 shows some glasses and their relative strength in generating fluorescence light for an excitation at the wavelength $365 \mathrm{~nm}$. The autofluorescent light spectrally overlaps with the sample related signal and cannot be filtered easily. In an ideal objective for fluorescence microscope, materials have very low autofluorescence level ${ }^{13}$. The choice of a low fluorescent microscope objective is important for high sensitivity imaging. Sufficiently low fluorescent background has to be obtained for the detection of single molecules. Autofluorescence emitted by an objective has to be minimized in the design phase of an optical instrument.

*herbert.gross@uni-jena.de; phone +49 (0) 3641-947992

Optical Systems Design 2015: Optical Design and Engineering VI, edited by Laurent Mazuray,

Rolf Wartmann, Andrew P. Wood, Proc. of SPIE Vol. 9626, 962609 - () 2015

SPIE $\cdot$ CCC code: $0277-786$ X/15/\$18 $\cdot$ doi: $10.1117 / 12.2191260$

Proc. of SPIE Vol. $9626962609-1$ 


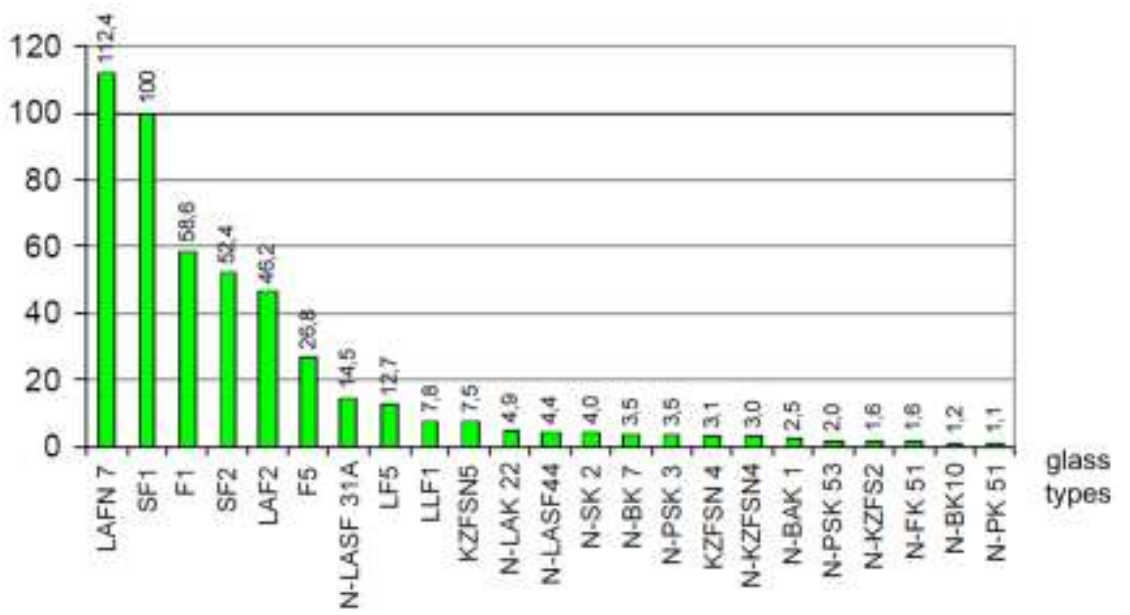

Figure 1. Relative fluorescence strengths of some Schott glasses, normalized on the value of SF1. The excitation is at $\lambda=$ $365 \mathrm{~nm}$, the signal is spectrally integrated ${ }^{12}$.

For simulations, commercially available raytracer allow to model autofluorescence in glasses as volume scattering. Large numbers of blue rays are traced through the objective, converting to red fluorescent rays with a certain probability. While the commercial software programs are relatively exact using real ray tracing and only few approximationsare made, they are often based on counting the number of rays on the detector. Immediately, issues with shot noise occur for too small numbers of rays. This makes these approaches rather time consuming and prohibits autofluorescence calculations during objective design. In this report, we develop a simple model for autofluorescence calculation based on coupling of excitation and fluorescent light in phase space. Tracing only few distinguished rays, we achieve a tremendous gain in simulation speed compared to the volume scattering models. Despite the approximations made, we find good agreement with results from real raytrace models.

\section{The proposed model}

Conventional calculation schemes are using simple raytrace on the basis of a Monte-Carlo approach to count for those rays of the excitation source, that reach the detector. The area and the solid angle of the light source is sampled with rays and finally the rays on every pixel of the sensor are collected. In modelling the process of fluorescence light generation, the Stokes shift of the wavelength from blue to red is taken into account as well as the isotropic radiation of the molecular re-emission. Furthermore the finite probability of the blue light absorption in the material under consideration is obtained by defining an appropriate free path length according to Beer's law. The problem if this kind of calculation scheme is the very small efficiency. Many rays have to be traced, before a signal is obtained with acceptable small statistical noise due to the random number selection of the rays. The corresponding long computational time prevents this simple brute force method to be used in optical design and system optimization. Therefore a different approach is needed to make the real calculation more efficient ${ }^{14}$. The main problem of the simple scheme described before is the small area and the small solid angle of light in the considered lens, that reach one pixel in the detector plane. The basic idea now is to separate the calculation into two steps and to calculate the small value of the coupling quasi-analytical. First the excitation light in calculated with the help of two paraxial marginal rays limiting the light cone, starting from the object plane until the lens. For simplicity, transmission illumination and only one illumination point on axis is considered. Figure 2 shows this part as the blue light cone in one transverse dimension y. In the lens, the intensity of the excitation is evaluated by basic photometric formula with the help of the ray data. Now the generation of the fluorescent light into an isotropic solid angle of the size $4 \pi$ takes place, this is illustrated in green in the drawing. Only those rays coming out of the illuminated cross section area in the corresponding solid angle reach one pixel with size $\Delta y^{\prime}$ of the detector, the corresponding light pipe is sketched in the figure in red. This small portion of light is calculated effectively not by probing forward propagating rays but by backwards calculation of rays from the pixel area in the image plane. Due to the image sided acceptance aperture angle of the system and the size of the pixel, a small overlap with the excited fluorescence light is observed. 


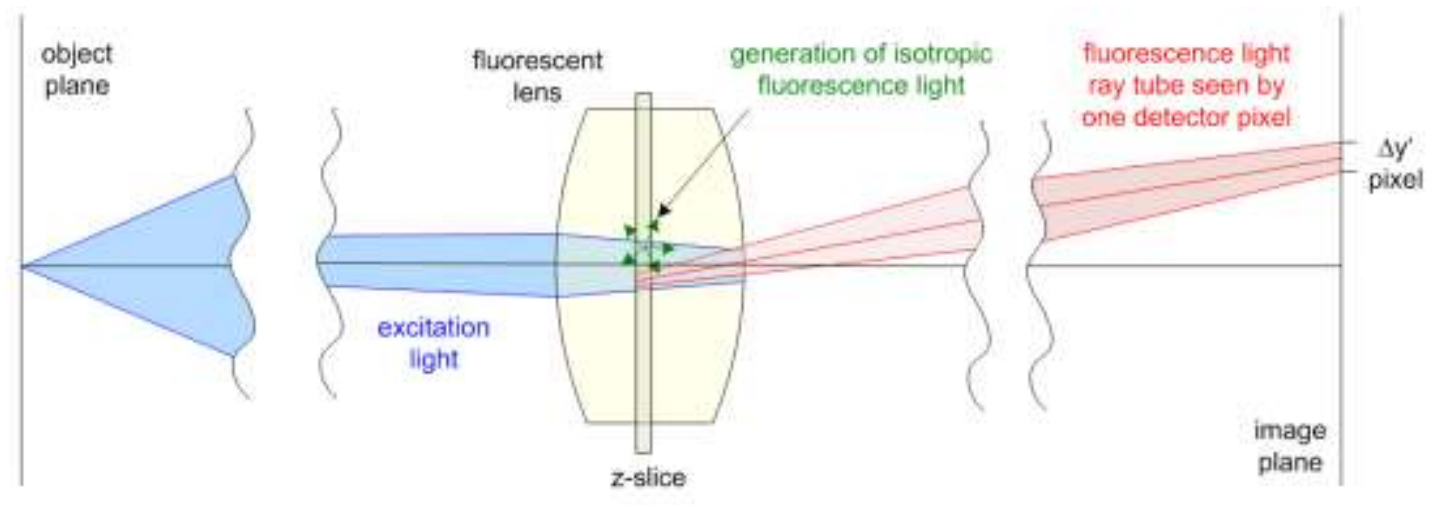

Figure 2. Schematic setup of the model with excitation light coming from the object plane, total fluorescent light created in the lens and detected fluorescence light reaching one detector pixel.

The poor geometrical coupling efficiency between the excited light cone and the detectable light tube by one pixel in the lens is approximated by a simple scheme. If the lens is discretized in z-slices, in every thin element the y-coordinates can by considered to be constant. It is convenient to illustrate the rays and ray bundles in the phase space, that is formed by the lateral coordinate and the angle in one plane $\mathrm{z}^{15}$. The ray bundles are described by corresponding areas, which have the shape of parallelograms. This is illustrated in figure 3 . The boundaries of the parallelograms can be obtained by tracing a small number of edge rays, which is quite fast.

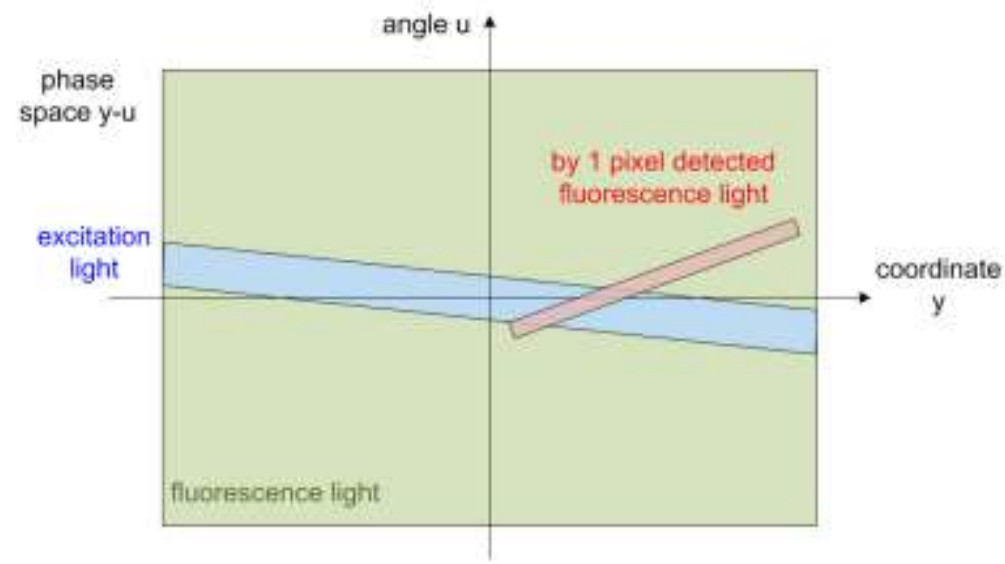

Figure 3. Sketch of the spatial and angular overlap of the excitation (blue), fluorescence (green) and detected (red) light in the phase space. Every ray bundle corresponds in one transverse dimension to a specific shaped area. The efficiency of transfer is given by the common area, which is seen in red here.

The efficiency is now a product of several factors, the most critical one is this area overlap, which is calculated geometrically in the phase space. If only a centered pixel is considered and constant factors are collected in a normalization constant $\mathrm{N}$ and the usual case is considered, that the ray bundle diameter of the excitation is larger than the observation bundle diameter, the transfer efficiency of autofluorescence light is given by

$$
P=N \cdot \eta_{\text {fluo }} \cdot \sum_{j}^{\text {planes }} \Delta z_{j} \cdot \Delta u_{\text {edge }, j}^{2} \cdot \frac{y_{\text {edge }, j}^{2}}{y_{M R, j}^{2}}
$$

here $\Delta \mathrm{z}_{\mathrm{j}}$ is the width of the slice no. $\mathrm{j}, \Delta \mathrm{u}_{\text {edge,j }}$ is the angle difference of the edge ray bundle for one pixel, $\mathrm{y}_{\mathrm{MR}, \mathrm{j}}$ is the excitation ray bundle diameter in the slice and $y_{\text {edge,j }}$ is diameter of the edge ray bundle from the detector an $\eta_{\text {fluo }}$ is the physical efficiency of autofluorescence light creation. The last two terms describe the geometrical area overlap in the phase space as explained before. In this formula, the sum represents the total geometrical factor of the transfer efficiency of the lens, the pre-factor the physical parameter of the material. 
If the model is considered in more detail, a serious photometric analysis of the sizes of areas and angles is necessary, but this is quite elementary. In three dimensions, the solid angles must be considered. The corresponding edge rays of the ray bundles are determined by aiming paraxial rays, which are limited by the lens diameters and stop sizes. Here different cases must be distinguished depending on the location of the lens under consideration relative to the stop position. In particular, near to the object plane the maximum angle of the excitation light cone is determined by the illumination system and not by the observation pupil. Rays, that are usually not able to pass the pupil in the observation path can reach the detector due to the change in direction during the scatter event. In addition, vignetting effects of skew rays are taken into account by scaling the paraxial ray heights to real sizes of the lenses. The used approximations are paraxial raytrace, a finite discretization of the lens volume assuming constant ray height inside every slice, only one wavelength for excitation and fluorescence light respectively. The determination of the number of z-slices depends on the thickness of the lenses and the largest ray angles inside the lens volume. If the shape of the lenses is more complicated with strongly curved surfaces or additional chamfer, the size of the considered slices is modified correspondingly for every index $\mathrm{j}$. Furthermore a depletion of the excitation light, coating effects and absorption are neglected and all lenses are considered separately. From practical experience it is seen, that all these simplifications are of nearly no influence. Their impact on the accuracy of results is smaller than the uncertainty of the necessary fluorescence coefficients of the used glasses. In the practical applications thus mostly relative calculations are performed.

It is found in practice, that with a simple implementation on the basis of Matlab for the photometric calculation and raytrace the increase in speed for typical microscopic lenses are in the range of $10^{4}$. This enables the optical designer to incorporate this kind of false light determination into a merit function for optimization. It gives the opportunity to select especially objective lenses, which shows a particular low intensity underground level due to autofluorescence of the glasses.

\section{Results}

To test the new implemented model, a simple microscopic lens is chosen. To demonstrate the relevance of the distance of the components from the object plane, a setup is modified with synthetic large oil immersion layer. The system has a numerical aperture NA $=0.5$ with magnification of 40 for a common tube lens and is build of 8 components, including cover glass and oil space. Figure 4 shows the basic setup together with the used materials. Since there are no autofluorescence data available for the oil TYPEA and the cover glass material K5, we assume here 1\% of SF1 for the oil and the same data as BK7 for K5.

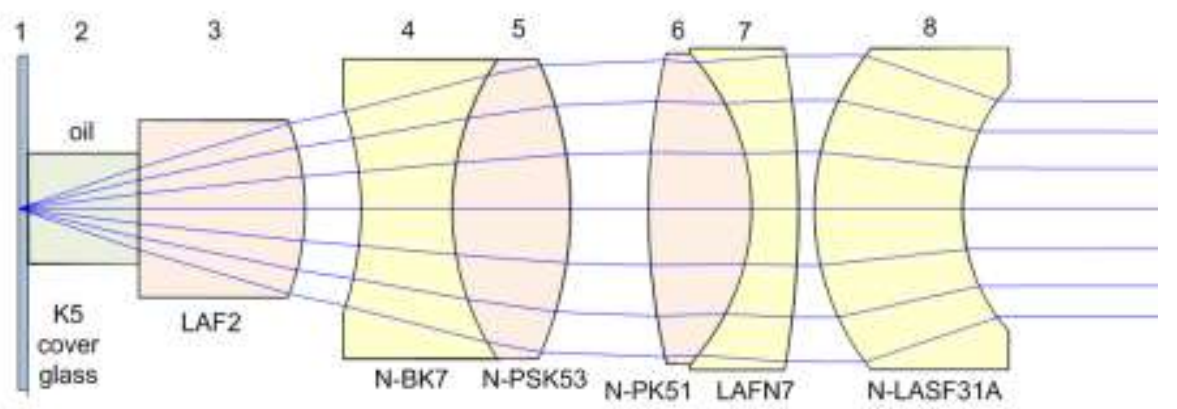

Figure 4. Layout of the immersion microscope objective with NA $=0.5$ and magnification 40 . The illumination light comes from left to right and is focused on the biological sample on the left surface of the cover glass.

Due to equation (1) it is obvious, that a component close to the object, which is then nearly conjugated to the detector, has a small value for $y_{\text {edge, } j}$ and therefore is very sensitive for autofluorescence stray light generation. The larger the distance from the object plane, the more uncritical is the behavior of the lenses from the geometrical point of view. The blue bars in figure 5 are the calculated contributions of the 8 elements in the system to the complete power of autofluorescent light. As a consequence of this result, a redesign of the system by changing glasses is performed. In particular in the critical lens No. 3 the Glass LAF2, which according to figure 1 is not very comfortable, is changed to NSK2. The resulting design therefore has a reduced problem with stray light by a factor of 3 , if cover glass and oil is excluded. The contributions are seen in figure 5 in red. It should also be mentioned, that the glass LAFN7 also has a large conversion coefficient. But due to the rear position inside the setup the overall effect can be completely neglected. 


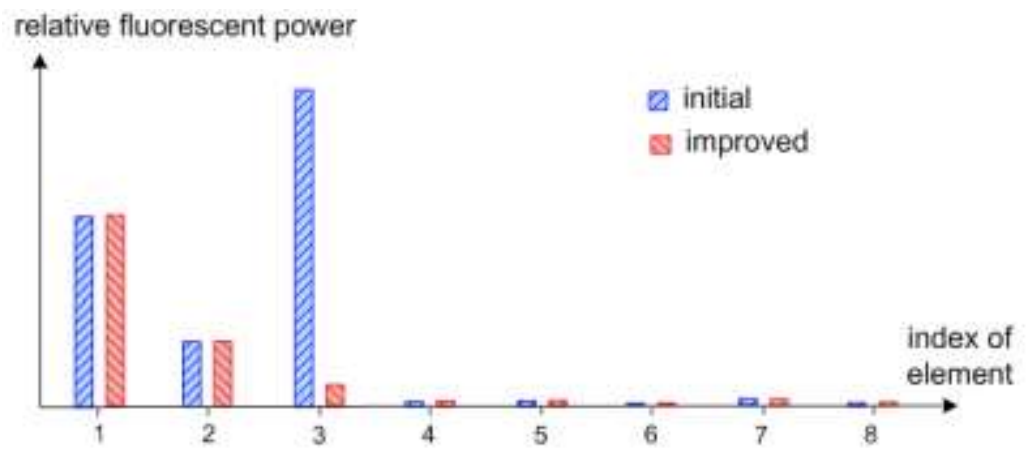

Figure 5. Fluorescent power of initial and improved microscope objective systems. After glass replacement the third element exhibit less autofluorescence.

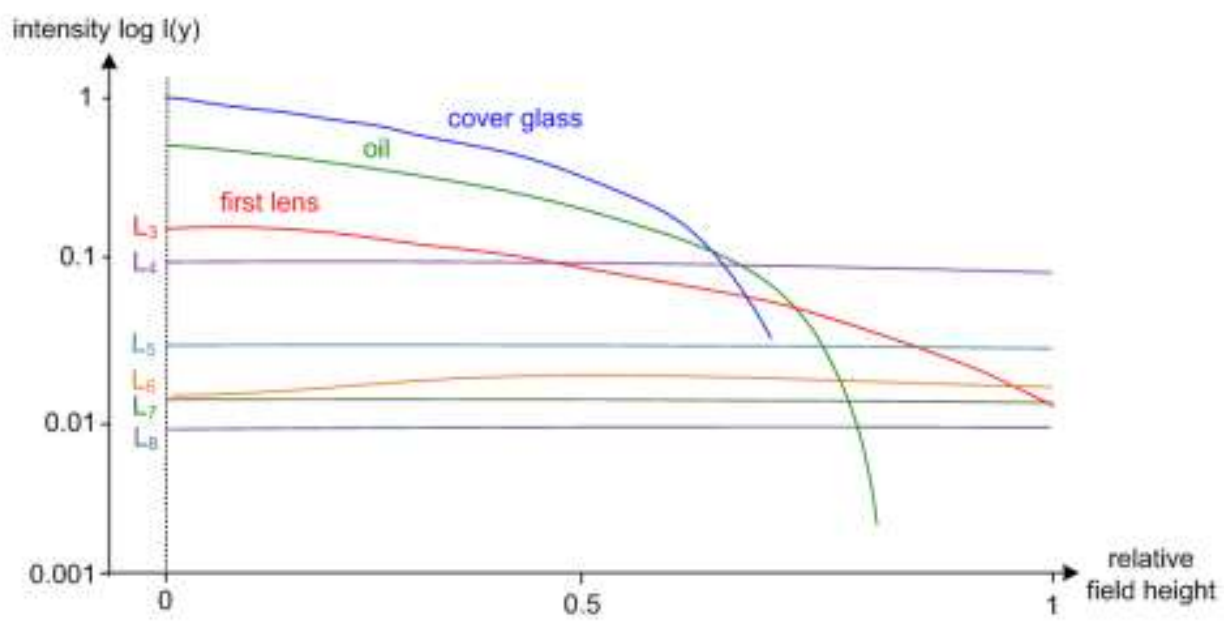

Figure 6. Autofluorescent power distribution on the detector for upper pixels (16 pixels of total 32) for all elements of the system.

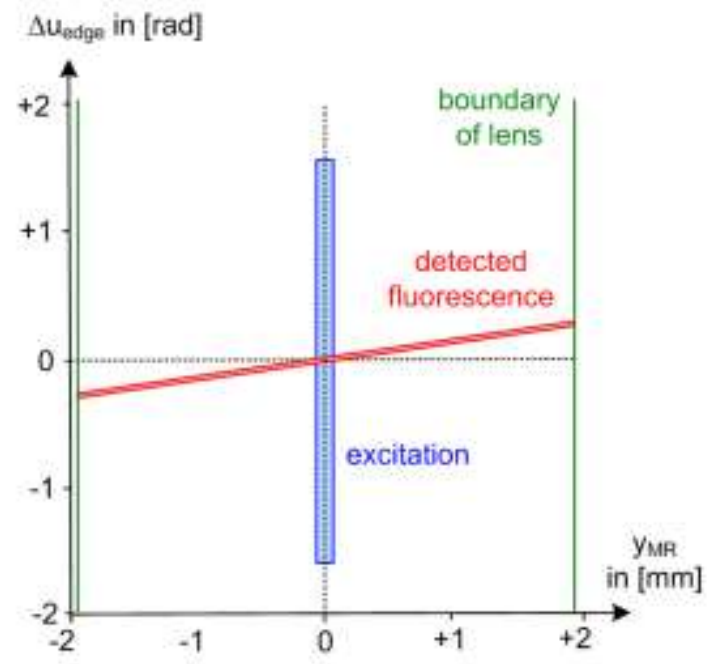

Figure 7. Phase space representation of the power coupling for the cover glass with nearly perfect pixel imaging 
Figure 6 shows the change of the autofluorescence signal as a function of the detector position for the improved design. It is seen, that a stronger fall off towards the edges of the detector is seen for the components close to the object plane. Obviously this stems from the near conjugate location of the secondary light source.

Figure 7 shows the real conditions of the power coupling area overlap in the phase space for this example in the special case of the cover glass. The nearly perfect imaging of the pixel generates a rather narrow parallelogram area for the detected light of one pixel. This again demonstrated the run time advantage of this quasi-analytical method of calculation.

\section{Evaluation of the new model}

To evaluate the properties and in particular to check the accuracy of the new model, test calculations are performed with Zemax, FRED and the new tool. The two commercial tools are used to model the autofluorescence light by volume scattering. Due to the different scaling and normalization and additional approximation, that are needed in the software tools, only relative results are comparable. The handling of the maximum numerical aperture angle was one of the critical aspects in this check calculation. In particular in the cover glass, in principle a full half space is illuminated by the point source in the object. A further problem stems from the sin-tan approximation in paraxial optics, which is used in our model. This must be restricted in the real calculation This aspect can be modelled in the new implementation in two different ways, one opportunity is to limit the numerical aperture to the nominal value, in the other case an unrestricted angle is allowed. From the manuals of the programs it can be seen, that different algorithms are used to calculate the scattering in the volume. This has the consequence, that the results of Zemax and FRED are differing by a factor of 3. Therefore we introduce relative coefficients $\varepsilon$ of the autofluorescence power contributions. These coefficients compare the efficiencies $\eta_{\text {fluo }}$ of two neighboring lenses. Figure 8 illustrates the results. It is seen, that all approaches are slightly different, but they are all in the same range, if a full angle is allowed in the new method. Larger differences are observed in the more critical front media, in particular for the oil space. For the real lenses the results are only changing by less then $20 \%$. The number of rays in the brute force simulations is selected to get a statistical uncertainty of smaller than 20\%. With these numbers Zemax needs 15 hours for the computation, FRED 17 hours and the new tool 7 seconds.

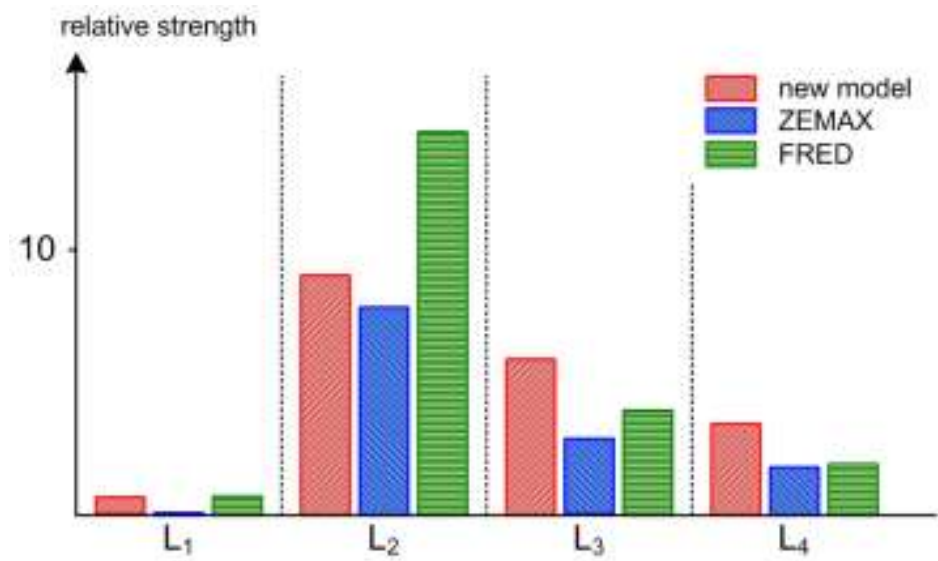

Figure 8. Comparison of paraxial 3D model results with commercial raytracer Zemax and FRED for the example system. The calculation with the paraxial model was performed for the cases of full NA.

\section{Conclusion}

Analytic 3D paraxial model was developed and implemented to perform autofluorescence analysis in microscope objectives. The model allows for fast analysis of optical systems considering both geometric factors and material conversion efficiency. Contributions of all elements can be analyzed. Different systems can be compared with each other. Influence of replacing glasses is investigated. The model can be used to investigate and improve an existing microscope objective to decrease autofluorescence background in the design phase. The results of the 3D model were verified with commercial software where autofluorescence was modeled as a bulk scattering. The model results are in the same range of accuracy as volume scattering methods. Tracing only few rays, we obtain gain in computation speed of a factor of four orders of magnitude comparing to commercial optical design software. 


\section{References}

[1] J. B. Pawley, "Handbook of biological confocal microscopy," New York, Springer, 2006, pp. 442-444.

[2] M. Fricker, J. Runions and I. Moore, "Quantitative Fluorescence Microscopy: From Art to Science," The Annual Review of Plant Biology, no. 57, pp. 79-107, 2006.

[3] P. D. Andrews, I. S. Harper and J. R. Swedlow, "To 5D and Beyond: Quantitative Fluorescence Microscopy in the Postgenomic Era," Traffic, no. 3, pp. 29-36, 2002.

[4] K. R. Spring, "Fluorescence microscopy," Encyclopedia of Optical Engineering, 2003.

[5] V. L. Mosiman, B. K. Patterson, L. Canterero and C. L. Goolsby, "Reducing Cellular Autofluorescence in Flow Cytometry: An In Situ Method," Communications in Clinical Cytometry, no. 30, pp. 151-156, 1997.

[6] N. S. Wang and M. Simmons, "Effect of background fluorophores on the NADH Fluorescence probe signal," Biotechnology techniques, vol. 5, no. 4, pp. 241-246, 1991.

[7] M. Neumann and D. Gabel, "Simple Method for Reduction of Autofluorescence in Fluorescence Microscopy," Journal of Histochemistry \& Cytochemistry, vol. 50, no. 3, pp. 437-439, 2002.

[8] T. J. Staughton, C. J. McGillicuddy and P. D. Weinberg, "Techniques for reducing the interfering effects of autofluorescence in fluorescence microscopy: Improved detection of sulphorhodamine B-labelled albumin in arterial tissue," Journal of Microscopy, vol. 201, no. 1, pp. 70-76, 2001.

[9] N. S. Wang and M. B. Simmons, "Fluorescence modeling in a multicomponent system," Biotechnology and bioengineering, vol. 38, no. 8, pp. 907-922, 1991.

[10] C. H. A. Van de Lest, E. M. M. Versteeg, J. H. Veerkamp and T. H. van Kuppevelt, "Elimination of Autofluorescence in Immunofluorescence Microscopy with Digital Image Processing," The Joumal of Histochemistry and Cytochemistry, vol. 43, no. 7, pp. 727-730, 1995.

[11] J. S. Stroud, "Optical Absorption and Color Caused by Selected Cations in High-Density, Lead Silicate Glass," Journal of the American Ceramic Society, vol. 54, no. 8, pp. 401-406, 1971.

[12] SCHOTT AG, "TIE-36: Fluorescence of optical glass," 2010. [Online]

[13] J. W. Lichtman and J.-A. Conchello, "Fluorescence microscopy," Nature methods, vol. 2, no. 12, pp. 910-917, 2005.

[14] O. Rodenko, "Autofluorescence modeling in microscopy", Master Thesis, University Jena, 2015.

[15] M. Testorf, B. Hennely, J.Ojeda-Castaneda, "Phase Space Optics-Fundamentals and Applications", McGraw Hill, 2009 